\title{
Integrating information literacy in IT courses: Information technology students' perceptions of embedded librarians
}

Scott Spangler, Middle Georgia State University, USA, scott.spangler@mga.edu

\begin{abstract}
The purpose of this pilot study is to assess undergraduate Information Technology (IT) students' perception of benefits from having an embedded librarian in an online learning management system. The pilot research data were collected through a random sample of volunteer focus group members after course completion. Similarly, to the literature methodology, the researcher shadowed a post-course focus group interview session to seek congruent literature themes: perception of comfort in experience, the perception of assignment confidence, and enhanced perception in research abilities. The data were first spiraled down using Quirkos qualitative analysis software and discussed to form a grounded theory. The pilot study concurs with prior literature's three main constructs: comfort, confidence, and recognition. Additionally, this study recognizes four additional themes: helpful, recognition of embedded librarians, interpersonal growth, and self-efficacy. Finally, this study acknowledges its limitations and recommendations for future research.
\end{abstract}

Keywords: Embedded librarian, course-integrated instruction, online instruction.

\section{Introduction}

The rise of online education has significantly modified how curriculum distribution and administration has changed students over the last decade. The University System of Georgia (USG) instituted a learning management system (LMS) to support and aid student success and curriculum instruction statewide in 2013. Currently, the USG employs the LMS Brightspace/D2L "via a centrally-hosted integrated learning environment that hosts more than 100,000 courses and supports more than 300,000 users" to facilitate asynchronized, online, and hybrid courses throughout all of its institutions (University System of Georgia, 2019, para. 1). Online or asynchronized instruction transpires when students are geographically separated from traditional course classroom situations and receive information through computer assistance. Student learning on LMS platforms are "independent of time and place" and engage in lessons separate from real-time instructions (Mayadas, 1997, p. 2).

USG students can access course materials when and where needed regardless of time and situational space. Course shells are available for all instructors to develop content, learning objectives, share and disseminate classroom materials, and engage in collaborate discussions or coursework through informal learning compositions (University System of Georgia, 2019). Asynchronized instruction encompasses learners (students) participating in computerassisted instruction (online learning) delivered through time and space by the Internet. Students 
are separated from traditional classrooms in a fulltime online environment or in a hybrid system that allows for more in-depth learning through asynchronized tools. Additionally, instructors can create scalable learning designs to specifically suit the needs of its disciplinary populations (Matlin \& Lantzy, 2017, p. 97). The asynchronized or hybrid learning environments circumnavigates the challenges college students face today like "juggling classes, assignments, and projects while getting the help and assistance needed to be successful academically" (Millsaps, 2013, para. 1). LMS provide students with a centralized location on the web to access course materials. Students have real-time access to course material that circumnavigates accessibility and disabilities issues. Additionally, it allows students to integrate with geographically separated peers and instructors. USG facilitates more than 160,000 courses that are all accessible on all student computer platforms or smart devices connected to the Internet (Millsaps, 2013, para. 10). The LMS offer instructors the ability to create extensive tutorials (content) that can be viewed at a self-paced administration before it becomes outdated (Mestre, 2012; Zang, Watson, \& Banfield, 2007).

Colleges and universities recognize $60 \%$ of students access to research materials through mobile devices. Users' (students) demand digital content on laptops, tablets, and smartphones to enhance learning especially with library resources (Dahlstrom \& Bichsel, 2014; Tumbleson, 2016, p. 227). With the innovation drive afoot, librarians are attempting to move out of the traditional brick and mortar setting and provide services virtually. Students are accustomed to fingertip virtual assistance from faculty-hence it is a natural progression for librarians to create a virtual venue for collaboration (Paganelli \& Paganelli, 2017, p. 55). Clark and Chinburg (2010)'s research analyzed student citation use between face-to-face and an online course with tutorials embedded in the course shell. They found no significant differences between delivery methods. Students' literacy of library resources were not affected through online digital books or PDFs. With no significant difference discovered, they stated, "embedded librarian process is providing the online students with similar support as the traditional face-to-face students receive" (Clark \& Chinburg, 2010, p. 538). Hence, early literature findings on embedded librarians in a hybrid course shadowed students expressing a greater perception of comfort in experience, perception of assignment confidence, and enhanced perception in research abilities from the new paradigm (Edwards, Kumar \& Ochoa, 2010).

\section{Embedded Librarians}

The concept of embedded persons is not unique to academia. The term embedded derives from the journalistic correspondent action where reporters are entrenched in combat zones with troops (Kesselman \& Watstein, 2009). In this embedded case, the librarian becomes a constant collaborator for students and instructors in the face-to-face, hybrid, and virtual spaces to expand contemporary library services (Dewey, 2004, p. 5). The librarians embedded directly into online course shells essentially become "extreme liaisons" and faculty collaborators to foster student achievement (Edwards et al., 2010, p. 273). Edwards et al. (2010)'s research asserts that the embedded librarians become key factors in student research development similar to Kesselman and Watstein (2009)'s findings.

Zang, Watson, and Banfield (2007) studied computer-assisted library instruction and revealed that the collaborators aided students in achieving the learning outcomes. The literature points out 
early pilot programs integrated librarians in face-to-face classrooms to accommodate hands-on learning activities and to understand the value of prolonged information literacy instruction. In this situation, librarians facilitated instructional sessions and lectures to aid students' in critically evaluating sources for assignments. Inaugural research studies on the perceptions of face-to-face embedded librarians uncovered that both faculty and students who are collaborating with the liaisons were satisfied with outcomes. Although, students in both pilot studies stated they forgot their librarian availability if the liaisons stopped being physically present (Dugan, 2008; Hall, 2008). With the ever-changing virtual academic sphere, embedding librarians into online courses "is a natural extension of the concept of embedding librarians into face-to-face course" to mitigate geographical distance disputes in educational programming (Edwards et al., 2010, p. 277). Nevertheless, the instructional methodology, the literature points out strategic collaboration between a faculty member and an embedded librarian is the foundation or core success factor in developing the liaison programs (Bozeman \& Owens, 2008; Chesnut et al., 2009; Edwards et al., 2010; Mathew \& Schroeder, 2006; Stewart, 2007).

To further understand the program's strengths and weaknesses, Edwards et al. (2010) conducted a pre and posttest survey on its population to understand if students' perceived notions of the embedded librarian program truly constructed greater essences of self-efficacy and confidence. The scholars' findings portrayed a significant percentage increase comfortability in researching abilities (44.4\% to $71.5 \%$ ) and a $16 \%$ increase in student confidence overall from working with librarians (p. 282). Students' qualitative responses uncovered positive reflections with working with embedded librarians. Particularly, the participants highlighted how integrated librarian tools increased their assignment success, abilities, significantly built overall confidence with researching. Data demonstrated a $16.6 \%$ increase in confidence in completing the task, $23.9 \%$ increase in comfort with working with library resources, and a $27.1 \%$ increase in comfort working with databases because of supplementary videos and information provided by the embedded librarians (Edwards et al., 2010, p. 284). Xiao (2010)'s research ( $\mathrm{n}=71$ ) parallels Edwards et al. (2010) survey data, and concluded that students who received library orientation programs and supplements state the resources are helpful (92\%), directed them to discover specific course resources (90\%), and improved their research skills (83\%). A later posttest in the research $(n=32)$ found similar findings with an increase $3 \%$ overall and an additional discovery of $96 \%$ of the students remarking the program helped them to reduce citation errors or plagiarism.

Other scholars mirrored the findings for face-to-face and online embedded librarians' collaboration. Particularly, the literature portrays themes of increases in library awareness, confidence in abilities, and citation clarity. Later studies uncovered self-efficacy, anxiety and confidence building through the utilization of embedded librarians (particularly with online or distance students). Students commented-beyond confidence and increased research skills abilities-online librarians and coursework tools increases their connection to the university and opportunities to find critically discipline-specific relevant citations. In the post-course survey, students reflected a significant difference $(0.71)$ on all accounts with increased confidence, success, abilities, citation knowledge, and reduced anxiety (Kumar \& Edwards, 2013). Other scholars' research reflected similar findings of student increases in confidence, anxiety reduction, and self-efficacy assurances (Blake et al., 2016; Horn, Maddox, Hagel, Currie, \& 
Owen, 2013; Matlin \& Lantzy, 2017; Pederseon-Summey \& Akers-Kane, 2017). Although when comparing face-to-face instruction versus online tutorial instruction in 2017, researchers from California State University, San Marcos (Matlin \& Lantzy, 2017) did not find a significant difference between two separate student populations. But the researchers stated both groups did portray a higher level of confidence.

Similarly, other research demonstrates students, who use online tutorials, present slightly better assignments than in-person instruction-only students (Matlin \& Lantzy, 2017, pp. 102-104). In 2017, they discovered a congruent finding with historical literature. By tracing four-year graduation rates, researchers uncovered students, who were subjected to library instruction in face-to-face and embedded programs at least one time in four years of study, had an increased GPA and increased chance of graduating in four years, and have a stronger understanding of barriers to graduation success (Bowles-Terry, 2012; Soria, Fransen, \& Nackerud, 2017).

\section{METHODOLOGY}

Upon approval of the Middle Georgia State University's Institutional Review Board (IRB), a focus group methodology was adapted with permission from Edwards et al. (2010) model to explore experienced meanings behind the phenomena. The purpose of this study is to validate the literature's findings and construct a conceptual grounded theory model to understand the central phenomenon (participants' experiences with embedded librarians). First, this study sought to determine if students' perceptions of online embedded librarians have congruent findings with the literature constructs: perception of comfort in experience, the perception of assignment confidence, and enhanced perception in research abilities. Secondly, this research utilized Lingelbach (2018)'s grounded theory approach to interviewing participants to discover if their perceptions on embedded librarians in online course LMS has changed or formed new impressions.

\section{Procedure and Research Design}

The researcher created a post-course focus group of volunteer participants ( $n=7)$ upon IRB approval. The design of the research followed Lingelbach (2018)'s model and Edwards et al. (2010)'s model. The design started with the construction of an IRB approved script, research question, and participant consent form based on Edwards et al. (2010)'s model. The research questions generated through the literature and its gaps with permission. Participants were read a confidentiality statement and instructed on the ability to stop the research at any time. All participants signed an IRB approved consent before starting the interviews. Additionally, the researcher utilized Lingelbach (2018)'s open-ended qualitative interview sessions model to collect and validate the data. Furthermore, to ensure data validation and bias, the researcher interviewed participants individually. During the interviews, the researcher asked participants to elaborate on the research questions' answers if they stated a keyword in the literature's findings such as confidence, comfort, helpful, or self-efficacy similar to Lingelbach (2018)'s open-ended data collection method. The open-ended questions provided participants an opportunity to elaborate on emerging patterns and themes instead of using "numerical data studies that would limit the study in strict boundaries or parameters" (Lingelbach, 2018, p. 33). Lingelbach (2018)'s grounded theory model allowed the researcher to construct an "analytical sense of meanings and 
actions" (p. 34). To accomplish the data construction, the researcher transcribed the interviews and then uploaded the transcriptions into Quirkos Software for coding. Data analysis included a coding process through Quirkos software. The software creates through its discovery "Quirks" or data points of consideration. The Quirks are spiraled down in axial coding methods into larger and more organized Quirk themes that form the traditional grounded theory. The researcher utilized Lingelbach (2018)'s constant-comparative methodology to investigate phenomena and determine outliers in the data. Lingelbach (2018)'s transcription process, validation, and analysis method were used to construct the data's meanings and form a grounded theory. Additionally, the researcher followed Lingelbach (2018)'s method of providing the transcript to the participants to ensure data and findings validations.

\section{Sample}

The volunteer participants were $28 \%$ female and $72 \%$, male and recruited through Edwards et al. (2010) purposeful sampling method to maintain consistency. The commonly used purposeful sampling method in qualitative research selects participants based on the need of the study and ease of finding participants that meet the criteria (Lingelbach, 2018, p. 36). Similarly, to Edwards et al. (2010), the research criteria shadowed a post-course focus group interview session to examine the paradigm of embedded librarian assistance and LibWizard tool constructions in a hybrid classroom to validate instrument further. All students in the course had full access to an embedded librarian and LiWizard tools through the Brightspace LMS. About $72 \%$ of the participants stated they were traditional college students coming straight out of high school or a two-year junior college and about $28 \%$ were non-traditional students that are returning for a degree or in the workforce. The majority of participants $(43 \%)$ work full-time, and $29 \%$ work part-time and $28 \%$ stated having no employment while in college.

\section{Reliability and Validity}

The researcher further validated the data's credibility, authenticity, and accuracy of the findings following Lingelbach (2018)'s triangulation methods. The researcher then utilized Quirkos software to organize the transcriptions. Then open Quirk (nodes) codes were created to discuss and ensure trustworthiness, authenticity, and credibility. Following Lingelbach (2018)'s methods, axial coding was next engaged to connect the conditions and meanings. Finally, Lingelbach (2018)'s method of selective coding and visualization of the data was constructed in Quirkos Software to perform a model or theory. The analysis methodology allowed the researcher to concur with the literature visually, understand the outlier conditions, and present follow up proposition or conclusion (Lingelbach, 2018, p. 39).

Additionally, participants were allowed the opportunity to view their transcripts and constructed themes to ensure credibility, authenticity, dependability. To validate the data analysis' themes, the researcher discussed the findings with participants to ensure trustworthiness and confidence. The confidence in the data's construction provided the researcher validity in the meanings and that the findings were transferable to other members. With this in mind and permission from the original scholars' literature, the researcher formulated the research questions:

RQ1: Does the presence of an online embedded librarian affect student's perceptions of course comfort? 
RQ2: Does the assistance of an online embedded librarian's LibWizard tools affect student's perception of assignment confidence?

RQ3: Does the presence of an online embedded librarian create an enhanced perception of abilities from the presence of an embedded librarian?

\section{RESULTS}

The data spiral demonstrates some 238 independent Quirks (coded points) of assessment from the seven volunteer participants (Figure 1).

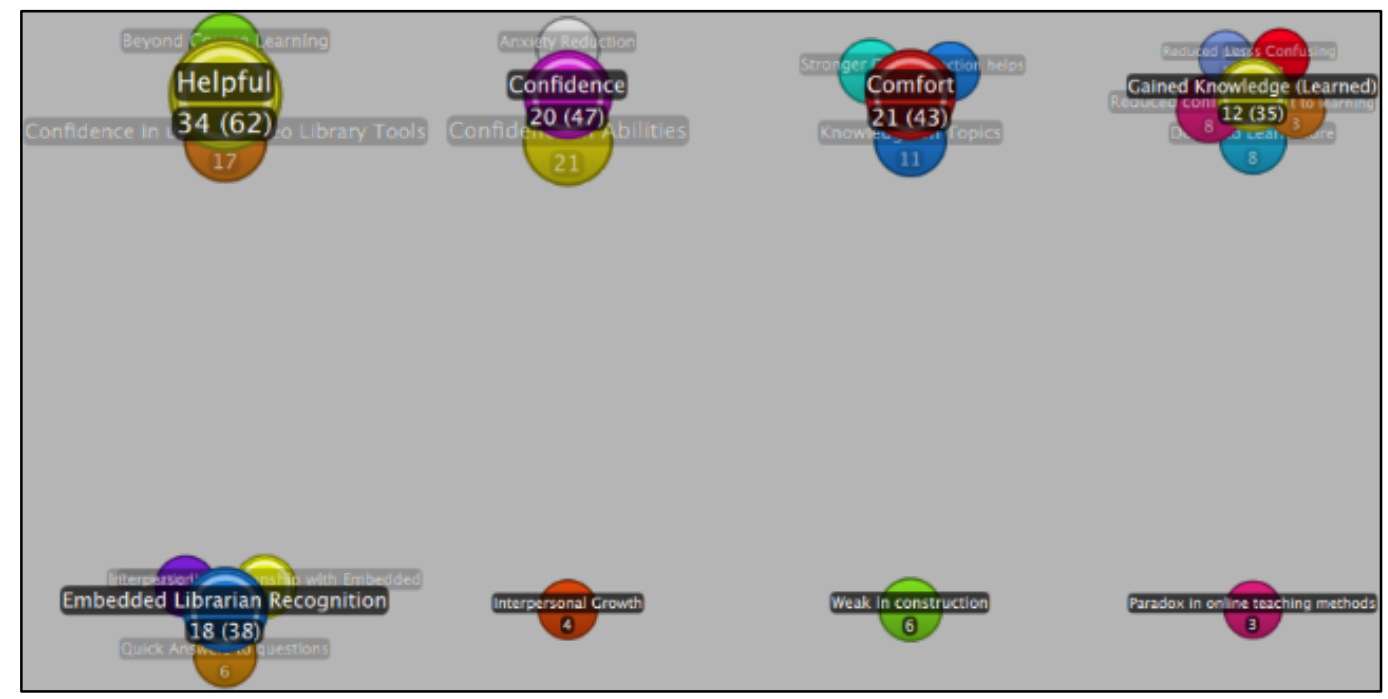

Figure 1. Quirkos Catalog of Themes

The analysis recognized eight primary parent themes: helpful, gained knowledge (learned), comfort, confidence, embedded librarian recognition, interpersonal growth, weak in construction, and paradox in online teaching methods. Overall the data construction accepts RQ1. The data represents 43 coded Quirks of participants gaining and having a perception of comfort from having an embedded librarian in the online course. Participant six stated:

She corroborated with me to get resources for my papers in the project management course and others I was taking. She helped me. So, I was able to get all the information I needed and [I was] able to email her directly with additional questions for help. I need that one-on-one time. In the library, their minds are everywhere and not focused on you. But through the [online course embedding] in the class, I was able to use her directly for a variety of things and get the one-on-one help I need because of my disabilities. I used her in all of my classes [despite being only embedded in the one course].

Participant seven reflected similar comforts:

You could tell it's an actual librarian there in the course and not just a search engine on the toolbar. It was a real person! The librarian's search tools [LibWiards] didn't just pull up random things. You were pulling up actual things that you're looking for and not just 
similar words. When I used it [Libwizard tools] instead of coping with other search tools [Galileo search] and praying it was headed in the right direction. It is comforting to know you're in the right direction when you have a research tool that points and directs you in the right direction.

Interestingly noted, the data constructed three sibling themes in the parent theme of comfort: knowledge of issues, stronger at citations, and topic direction help. Participants remarked 11 times finding their knowledge of topics was stronger from the use of embedded librarian tools. Additionally, the tools created a greater sense of comfort with course challenges. Participant six remarked:

It's frustrating when you're trying to get help-and you log in, and the student success center is too booked. So, she [having an embedded librarian in the course] is a good outlet and help. I used her in other classes also-where she wasn't embedded. As an example, she helped me with my legal and ethical issues course. I knew what I wanted to write about a certain topic. But, there was no embedded librarian in the legal course. So, she helped me because of the relationship I built with her through the other professor's class.

Additionally, participants stated seven times they had a stronger ability to create correct citations and a higher comfort level citing material. Overall, all the participants felt more comfortable from having the additional librarian's directions for research material.

Answering RQ2, the data represented 47 coded Quirks to confirm the assistance of an embedded librarian's LibWizard tools positive effects on students' assignment confidence. The parent theme of confidence grew two secondary or child nodes: confidence in abilities and reduction of anxiety. Participant four stated the librarian's virtual presence and tools gave her a greater sense of confidence and abilities. Participant four stated:

There was a window that launched another window [inside the LMS] to learn about the assignment's topic from library sources. It was a massive confidence boost. People [students in the group project] just navigated to it [library resources] themselves without guidance. And it gave me the knowledge, or the ability, for me to know I'm using better sources. It's [resources found from the librarian's tools] not off Google and or something I pulled off the internet. All the [librarian's] citation tools are right there on the page [in the LMS course]. It's all there. It [the library tools] teaches us how to write, use, or go to the easy bib. It's all on the site [LMS course shell] for the resource. Not all the courses were like that. I'd say the LibWizard tools made it so, other tools were not needed. The embedded librarian and her tools built in [to the course] made it so easy. Everything was right there.

Additionally, the data analysis for RQ2 supported a second child theme. The participants remarked that working with embedded librarians and their tools caused a reduction in anxiety. This discovery was similarly uncovered in the historical embedded librarian literature (Kumar \& Edwards, 2013). Furthermore, participants made positive remarks about the embedded librarian's classroom tools having the ability to reduce stress and anxiety levels. Participant two remarked, "The tools [LibWizards] make the instructions clear, and it stops confusion. It also reduces 
anxiety about citations." Participant six elaborated deeper explaining, "It was a great strategy to release [sic] stress, and I know it's [everything is going to be] ok because you have somebody to help you. We have the student success center to go to, but this is nothing like them [student success center aids]. The embedded librarian had the time to actually help me and find resources and links to help me be a success." The child theme was observed in Kumar and Edwards's (2013) research and similarly noted in Edwards et al., (2010) despite not being definitively declared.

Answering RQ3, the data (38 Quirks coded) represented a positive correlation between the presence of an embedded librarian and students' enhanced perception of abilities. The findings are congruent with the literature (Edwards et al., 2010; Xiao, 2010; Horn, Maddox, Hagel, Currie, \& Owen, 2013; Kumar \& Edwards, 2013; Blake et al., 2016; Pederseon-Summey \& Akers-Kane, 2017). Additionally, the data uncovered four child themes inside the parent theme: interpersonal relationships, quick to answer questions, emphatic listening, and embedded librarian motivation. Participants remarked the embedded librarians were easy to work with and they enjoyed collaborating with the students. All participants remarked the embedded librarian's presence in the course created a feeling of comfort and level of success. Participant one remarked, "Seeing her face was nice. When you need help, you know who to look for in the course. It was easy to locate her in the course shell, and she also had her phone and email on the homepage. So, I could get in touch with her really easy." Participant six concurred:

She collaborated [with me] to get resources for my papers in my project management course. She helped me to get all the information I needed for each assignment. Additionally, I was able to email her directly for additional help or questions. Through the emails and help, I built a relationship with her. Before the course, I didn't know I could even get help! It [embedded librarian's help] made me learn more, and her help allowed me to write better papers. In turn, it allowed my reader or audience to learn more about the topic that I was getting at [in my papers I wrote for class].

\section{CONCLUSION}

Although much of the literature proposes students learn equally between face-to-face and asynchronized delivery, new research demonstrates increases in student success, selfefficacy, and confidence when combined with embedded library instruction (Silke, Perrault, Ladenson, \& Nazione, 2015). The data study uncovered congruent themes with scholars in the field (Blake et al., 2016; Edwards et al., 2010; Horn, Maddox, Hagel, Currie, \& Owen, 2013; Kumar \& Edwards, 2013; Pederseon-Summey \& Akers-Kane, 2017; Xiao, 2010). The researcher's data analysis confirms RQ1, RQ2, and RQ3 are congruent in the findings with the literature. The data's analysis suggests students-utilizing embedded librarians in online course shells-gain confidence, comfort, and a higher perception of abilities from the collaborative projects.

Interestingly noted, the data analysis found several grounded child themes. Two of the child themes ran congruent with all participants: anxiety reduction and relationship building from the onset of utilizing embedded librarians' tools. The data analysis also discovered several of the literature's important parent themes: helpfulness, knowledge gained (learned), interpersonal 
growth, and paradox in online teaching methods. The helpfulness theme (62 Quirks) generated two child themes: beyond course learning (11 Quirks) and confidence in utilizing Galileo search library tools (17 Quirks). And the second parent theme, knowledge gained (learned), noted 35 Quirks codes and five child themes: reduced assignment confusion (8 quirks); reduced confusion levels (all courses); the desire to learn more (8 Quirks); less course confusion (3 Quirks); and convenient to learning (3 Quirks). The most notable child theme that came from the data's analysis uncovered was the desire to learn more. Participants remarked-in the validation of the data process-the theme was driven through meaningful relationships built with the librarians. The personal attention and connections made while students worked on projects created an essence of self-efficacy and a desire to do more academically. Participant two stated:

I feel as if other students could use this tool tremendously. It [the embedded librarian program] should be in all courses-especially those requiring citations, resources, and looking up information in a library. Additionally, the Zotero tool and its lessons helped. I would like more information about how to use library tools and ones like Zotero. After using some of the tools, I wish we could have more intro LibWizards. I myself and other students were having problems understanding how to make correct citations until I used the tutorial. The tutorials could also be really helpful to students with no computer background.

\section{Furthering the Study}

The researcher understands the study's small population makes its ability to generalize the findings difficult. To further the research and know if the grounded theories uncovered are generalizable, researchers should extend the population regionally, nationally, and internationally. Additionally, because of the small population, three of the parent themes are limited scientifically without any literature backing the constructions. The researcher understands this limitation and suggests the need for further investigation to recognize the branches: interpersonal growth, the paradox in teaching, and weak construction. However, the researcher will acknowledge the need for changes in the embedded librarians' tools. Specifically, six of the participants stated a need to eliminate redundant material in the LibWizard tools. This finding was driven inside the weak construction theme. Participants explained once the information is learned, such as a preliminary Boolean search mechanics, it should be dropped from further tools found deeper in the course's construction. Although this is a negative construct, it has actual merits. The theme creates a grounded theory demonstrating that if an embedded librarian is placed in a learning management course shell, students will utilize the librarian and the provided tools. The theory highlights the fact that the majority of the participants learned the lessons and utilized the embedded librarian's tools.

Although much of the literature proposes students learn equally between face-to-face and asynchronized delivery, new research and this data analysis demonstrate increases in student success, self-efficacy, and confidence when combined with an embedded librarian program (Silke et al., 2015). To further this study, researchers should create a quantitative survey that is designed to measure the underlying themes found in this paper. Additionally, a quantitative survey could easily be disseminated through online tools. By increasing the population's size and expanding its geographical limitations, the study could have a greater validation with the 
literature's findings. Secondly, the larger population could provide deeper meanings about the perceived helpfulness of embedded librarian projects. Although the data construction in this pilot study did confirm the original themes in Edwards et al. (2010), the small population may not be significant enough to correlate a valid finding. And finally, the presence of an embedded librarian in an LMS course shell did generate positive themes about self-efficacy and reducing student research anxiety. But again because of the geographically limited study and size, the findings may not be valid. Again, the researcher suggests a larger population and mixed method survey to understand the child themes acceptance levels. Nevertheless, this pilot study did concur with past literature's themes and find new child theme theories. Participant six summarized the students' perceptions of embedded librarians stating:

Once you get the word out there about embedded librarians in courses, it will be a good deal. I would love a way to book her. I know as people learn about this, she [the embedded librarian] is going to be booked. I want to make sure I can have a way to book her! When she [the embedded librarian] sees me, she motivates me! She tells me 'You need help? You come to see me!' Just knowing she is there and I now have her as a resource is very, very helpful and motivating.

The field of library science is continually changing. The speed of technology creates many new avenues for information transmission. To meet the demands of the ever-changing computer and technology fluent student culture in higher education, librarians must be in a constant state of best practices continuity and flexibility. Thereby, researchers need to continually question the effectiveness of new programs and technology instituted in library programs. The new findings uncovered here open avenues for questioning how the embedded librarians' programs increase student self-efficacy and academic growth. In the future, researchers should examine the variables of this study against a graduate student population and innovations in the field.

\section{REFERENCES}

Blake, L., Ballance, D., Davies, K., Gaines, J., Mears, K., Shipman, P., ... Burchfield, V. (2016). Patron perception and utilization of an embedded librarian. Journal of Medical Library Association, 104(3), 226-230.

Bowles-Terry, M. (2012). Library instruction and academic success: A mixed-methods assessment of a library instruction program. Evidence Based Library and Information Practice, 7(1), 82-95. https://doi.org/10.18438/B8PS4D

Chesnut, M. T., Henderson, S. M., Schlipp, J., \& Zai, R. (2009). Value-added library resources \& services through blackboard. Kentucky Libraries, 73(1), 6-12.

Clark, S., \& Chinburg, S. (2010). Research performance in undergraduates receiving face to face versus online library instructions: A citation analysis. Journal of Library Administration, 50, 530-542.

Dahlstrom, E., \& Bichsel, J. (2014). ECAR study of undergraduate students and information technology. ECAR: Research Report. Louisville, CO. Retrieved from http://www.educause.edu/ecar 
Dewey, B. I. (2004). The embedded librarian: Strategic campus collaboration. Resource Sharing and Information Networks, 17(1), 5-17. https://doi.org/10.1300/J121v17n01_02

Dugan, M. (2008). Embedded Librarians in an ag econ class: Transcending the traditional. Journal of Agricultural \& Food Information, 9(4), 310-309.

Edwards, M., Kumar, S., \& Ochoa, M. (2010). Assessing the value of embedded librarians in an online graduate educational technology course. Public Services Quartley, 6, 271-291.

Hall, R. A. (2008). The "embedded" librarian in a freshman speech class. College and Research Libraries News, 69(1), 28-30.

Horn, A., Maddox, A., Hagel, P., Currie, M., \& Owen, S. (2013). Embedded librarian services: Beyond chance encounters for students from low SES Backgrounds. Australian Academic \& Research Libraries, 44(4), 235-250.

Kesselman, M. A., \& Watstein, S. B. (2009). Creating opportunities: Embedded librarians. Journal of Library Administration, 49(4), 383-400.

Kumar, S., \& Edwards, M. (2013). Information literacy skills and embedded librarianship in an online graduate program. Journal of Information Literacy, 7(1), 3-17. https://dx.doi.org/10.11645/7.1.1722

Lingelbach, K. (2018). Perceptions of female cybersecurity professionals toward factors that encourage females to the cybersecurity field (Doctoral dissertation). Nova Southeastern University, USA. (1056) Retrieved from NSUWorks, College of Engineering and Computing. Https://nsuworks.nova.edu/gscis_etd/1056.

Matthew, V., \& Schroeder, A. (2006). The embedded librarian program. EDUCAUSE Quarterly, 29(4), 61-65.

Matlin, T., \& Lantzy, T. (2017). Maintaining quality while expanding our reach: Using online information literacy tutorials in the sciences and health Sciences. Evidence Based Library and Information Practice, 12(3), 95-113.

Mayadas, F. (1997). Asynchronous learning networks: A Sloan Foundation perspective. Journal of Asynchronous Learning Networks, 1(1), 1-16.

Mestre, L. (2012). Designing effective library tutorials: A guide for accommodating multiple learning styles. Oxford, United Kingdom: Chandos Publishing.

Millsaps, J. (2013, April 16). Desire2Learn learning management system helps students manage college classes communications. University System of Georgia, USA. Retrieved from https://www.usg.edu/news/release/desire2learn_learning_management_system_helps_stu dents_manage_college_class

Paganelli, A., \& Paganelli, A. (2017). The online embedded personal librarian approach to providing reference services via a course management system. Journal of Electronic Resources Librarianship, 29(1), 54-60. https://doi.org/10.1080/1941126X.2017.1270105

Pederseon-Summey, T., \& Akers-Kane, C. (2017). Going where they are: Intentionally embedding librarians in course and measuring the impact on student learning. Journal of 
Library Information \& Information Services in Distance Learning, 11(1-2), 158-174. https://doi.org/10.1080/1533290x.2016.1229429

Silke, K. J., Perrault, E. K., Ladenson, S., \& Nazione, S. A. (2015). The effectiveness of online versus in-person library instruction on finding empirical communication research. The Journal of Academic Librarianship, 41(2), 149-154. https://doi.org/10.1016/j.acalib.2014.12.007

Soria, K. M., Fransen, J., \& Nackerud, S., (2017). The impact of academic library resources on undergraduates' degree completion. College \& Research Libraries, 78(6), 812-823. https://doi.org/10.5860/crl.78.6.812

Stewart, V. D. (2007). Embedded in the Blackboard jungle: The embedded librarian program at Pulaski Technical College. Arkansas Libraries, 64(3), 29-32.

Tumbleson, B. (2016). Collaborating in research: Embedded librarians in the learning management system. The Reference Librarian, 57(3), 224-234.

University System of Georgia. (2019, January 18). GeorgiaVIEW, University System of Georgia, USA. Retrieved from https://www.usg.edu/georgiaview/georgiaview

Xiao, J. (2010). Integrating information literacy into Blackboard: Librarian-faculty collaboration for successful student learning. Library Management, 31(8/9), 654-668. https://doi.org/10.1108/01435121011093423

Zang, L., Watson, E. M., \& Banfield, L. (2007). The efficacy of computer-assisted instruction versus face-to-face instruction in academic libraries: A systematic review. Journal of Academic Librarianship, 33(4), 478-484.

\section{Author Biography}

Scott C. Spangler, D.Sc. worked as a photojournalist for over 20 years in the newspaper industry prior to receiving his doctorates at Robert Morris University. Currently, Dr. Spangler is an assistant professor of information technology at Middle Georgia State University's School of Computing and Mathematics. Prior to starting his tenure-track career at MGA, Dr. Spangler was a professor at Robert Morris University, Point Park University, Education Management Corporation's Art Institute of Pittsburgh, and Oakbridge Academy.

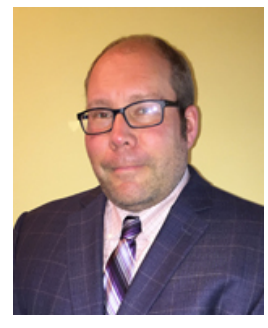

Spangler's doctoral ethnographic research centers around human-computer interaction and how cultures use innovations. His current research seeks meanings behind the digital culture's norms, actions, and meanings with technology. His most recent publications peer into the digital culture's understanding of privacy with mobile devices and how the digital culture uses innovations within everyday practices. Currently, Dr. Spangler is researching how the digital culture engages with online embedded librarians and their human-computer interaction tools. 